\title{
BMJ Open Burden of scrub typhus among patients with acute febrile illness attending tertiary care hospital in Chitwan, Nepal
}

\author{
Sangita Thapa (D) , ${ }^{1}$ Pradip Hamal, ${ }^{1}$ Navin Kumar Chaudhary, \\ Lokendra Bahadur Sapkota, ${ }^{2}$ Jaya Prasad Singh ${ }^{3}$
}

To cite: Thapa S, Hamal P, Chaudhary NK, et al. Burden of scrub typhus among patients with acute febrile illness attending tertiary care hospital in Chitwan, Nepal. BMJ Open 2020;10:e034727. doi:10.1136/ bmjopen-2019-034727

- Prepublication history and additional material for this paper are available online. To view please visit the journal (http:// dx.doi.org/10.1136/bmjopen2019-034727).

Received 03 0ctober 2019 Revised 19 June 2020 Accepted 30 July 2020

Check for updates

(C) Author(s) (or their employer(s)) 2020. Re-use permitted under CC BY-NC. No commercial re-use. See rights and permissions. Published by BMJ.

${ }^{1}$ Clinical Microbiology, Chitwan Medical College, Bharatpur, Nepal

${ }^{2}$ Department of Biochemistry, Chitwan Medical College,

Bharatpur, Nepal

${ }^{3}$ Department of Community Medicine, Chitwan Medical College, Bharatpur, Nepal

Correspondence to

Dr Sangita Thapa;

drsangitathapa@gmail.com

\section{ABSTRACT}

Objectives Scrub typhus is an emerging neglected tropical disease, reported from many parts of Asia including Nepal. This study aims to determine the seroepidemiology of scrub typhus among febrile patients attending Chitwan Medical College Teaching Hospital (CMC-TH), Bharatpur, Nepal.

Study design and setting This was a hospital laboratorybased prospective study conducted in CMC-TH (a 750-bed hospital) located in Bharatpur, Chitwan district of Nepal. Participants A total of 1797 patients visiting CMC-TH with acute febrile illness (temperature more than $38^{\circ} \mathrm{C}$ ) were enrolled in this study.

Methods A total of 1797 blood samples were collected from patients presenting with acute febrile illness. The samples were processed for detection of antibody for scrub typhus by ELISA for specific IgM antibody and WeilFelix test.

Results Out of 1797 serum sample of febrile patients, $524(29.2 \%)$ were scrub typhus positive. Maximum seropositive cases were from Chitwan district, 271 (51.7\%) with predominance among women, 314 (35.9\%). Scrub typhus was common among age group 51-60 years $(37.2 \%)$ and farmers, $182(37.8 \%)$. Highest seropositivity was found in July, 60 (57.7\%). Fever was common clinical symptom. Thrombocytopenia was seen in 386 (73.7\%) and raised transaminase aspartate aminotransferase, 399 (76.1\%) among seropositive cases. Weil-Felix test positive were $397(22.1 \%)$ and IgM ELISA positive were 524 (29.2\%). The correlation between IgM ELISA and Weil-Felix test showed statistically significant association $(r=0.319$, $\mathrm{p}<0.001$ ).

Conclusion High prevalence of scrub typhus implies that patients with acute febrile illness should be investigated for scrub typhus with high priority. There is utmost need of reliable diagnostic facilities at all levels of healthcare system in Nepal. Infection with scrub typhus was found high and this calls for an urgent need to introduce vaccine against scrub typhus. More sustain and vigorous awareness programmes need to be promoted for early diagnosis, treatment and control.

\section{BACKGROUND}

Scrub typhus, also known as tsutsugamushi disease, is an acute febrile illness caused by Orientia (Rickettsia) tsutsugamushi, a Gramnegative, obligate intracellular organism.

\section{Strengths and limitations of this study}

- A prospective study conducted in the largest tertiary care centre of Chitwan.

- For the very first time, seroepidemiology of scrub typhus was performed by Weil-Felix test and ELISA in Chitwan.

- The scrub typhus IgM ELISA is a flexible alternative to the indirect fluorescent assay technique.

- Comparison of commonly used rapid card test with ELISA was not done in this study.

- The gold standard test, indirect fluorescent assay, which was not used in this study, would have been the best indicator to find sensitivity and specificity of other tests.

The bite of a larva of trombiculid mites (chiggers) transmits the disease to humans. ${ }^{1}$ The mites are vector and reservoir of disease. The disease is transmitted by transovarian transmission in mite vectors. Human beings are accidental hosts. ${ }^{2}$ It presents from selflimiting, mild to fatal illness. Onset is characterised by fever, headache, cough, myalgia and gastrointestinal symptoms. The nonspecific signs and symptoms resemble other infectious diseases (enteric fever, dengue, malaria and leptospirosis). ${ }^{3}$

The burden of disease is reported from many parts of Asia including Nepal. ${ }^{4}$ Geographically, this endemic disease is confined to the regions of eastern Asia and southwestern Pacific (Korea to Australia) and from Japan to India and Pakistan. ${ }^{5}$ Every year a billion of people are in danger and almost a million of cases are reported from AsiaPacific region. ${ }^{6}$

Scrub typhus was first reported in Nepal as a cause of acute febrile illness in 1981 . Since then the disease has been endemic in different parts of the country. ${ }^{7}$ Major outbreaks of scrub typhus were reported from different districts of central Nepal after the 7.8 and 7.3 magnitude earthquake that 
struck Nepal on 25 April and 12 May 2015, respectively and subsequent aftershocks. ${ }^{8}$

In Nepal, scrub typhus has been documented from Nuwakot, Gorkha, Nawalparasi, Dhading, Sindhupalchowk, Kailali, Bara, Parsa, Dhanktuta, Sindhuli, Sarlahi, Ilam, Morang, Rukum, Makwanpur, Baglung, Sankhuwasabha, Bhojpur, Chitwan and other four districts according to Epidemiology and Disease Control Division. ${ }^{9}$ Although the disease is endemic, it is grossly underdiagnosed due to low index of suspicion by the clinicians, non-specific clinical presentation and lack of specific diagnostic facilities. ${ }^{10}$

During the wet season in dense areas of scrub vegetation, infected chiggers are especially found. Infected mites thrive in areas like grassy regions, forest clearings and riverbanks. The southern Terai region of Nepal is favourable environment for scrub typhus. ${ }^{11}$ Postearthquake outbreaks in various districts of Nepal might be a true reflection of altered environmental factors and epidemiological behaviour of the vector. It might be due to close proximity between humans and rodents in temporary shelters. ${ }^{12}$

Since different regions of Nepal appeared to be suitable hubs for scrub typhus, the clinical suspicion of scrub typhus in the differential diagnosis of fever of unknown origin is mandatory. Laboratory confirmation is essential to prevent and restrict the spread of the disease. Currently, serological tests are mainstay for diagnosis of scrub typhus. Among these, Weil-Felix test is the cheapest and easily available but unreliable test, while ELISA provides more sensitivity and specificity. Indirect immunofluorescence antibody is the gold standard. ${ }^{13}$ In Nepal, the burden of scrub typhus is underestimated due to lack of studies and availability of specific laboratory tests. Therefore, this study was undertaken to determine the seroepidemiology of scrub typhus among patients with acute febrile illness attending Chitwan Medical College Teaching Hospital (CMC-TH).

\section{MATERIALS AND METHODS}

\section{Study design and participants}

This was a hospital laboratory-based prospective study conducted in CMC-TH (a 750-bed hospital) located in Bharatpur, Chitwan district of Nepal from July 2016 to June 2017.

\section{Study population}

A total of 1797 patients visiting CMC-TH with acute febrile illness (temperature more than $38^{\circ} \mathrm{C}$ ) were enrolled in this study. After taking informed consent from the patients, a preformed questionnaire was used to collect the demographic and health-related information.

\section{Inclusion and exclusion criteria}

All patients with acute febrile illness of all age group attending CMC-TH were included in this study. Patients with acute febrile illness diagnosed with other associated infection such as dengue, leptospirosis, typhoid, brucella and malaria were excluded.

\section{Sampling procedure (blood sample collection, handling and transportation)}

A total of 1797 specimens were collected from patients attending CMC-TH. All the specimens were collected by veinipuncture aseptically with the standard operating procedure in BD Vacutainer, yellow colour coded and lavender color-coded tube from suspected cases of patients with acute febrile illness. Collection and transportation of specimens were done according to the manufacturer's instructions. Blood samples collected were received in the Department of Clinical Microbiology.

\section{Laboratory procedure}

Blood samples were tested for O. tsutsugamushi antibody. Immunological profiles like Weil-Felix test and IgM ELISA for detection of antibody were done according to the manufacturer's instruction. The profile of laboratory investigation of various laboratory parameters of all the patients was recorded.

\section{Weil-Felix test}

Patients with acute febrile illness were tested serologically for scrub typhus infection by Weil-Felix test for the qualitative and quantitative detection in serum (Tulip Diagnostic, India) according to the manufacturer's instruction.

In the three Proteus OX strains only, the OX-K was used in this study. The dilutions ranged from 1:20 to 1:1280. The highest dilution of sera showing visible agglutination was taken as the titre. A titre of 1:160 for OX-K was taken as positive for scrub typhus, this cut-off titre of this disease $\geq 1: 160$ of this test has been determined experimentally. ${ }^{14}$

\section{ELISA}

The scrub typhus IgM ELISA is a flexible alternative to the indirect fluorescent assay (IFA) technique. It has sensitivity and specificity of more than $90.0 \%$ for detecting specific antibodies. The antigen used in the wells was a recombinant $56 \mathrm{KDa}$-type specific antigen. The patient's sera at a dilution of 1:100 were tested using Scrub Typhus Detect IgM ELISA kits (InBios International, Seattle, Washington, USA) according to the manufacturer's instruction. The absorbance was read at $450 \mathrm{~nm}$ and the results recorded as positive or negative. The cut-off value of optical density (OD) was fixed at 0.51 following recommendations for determining the endemic cutoff titre in the kit protocol. The cut-off calculated from healthy volunteer was mean OD $(0.24)+3 \mathrm{SD}(0.09)=0.51$. We proposed a cutoff-OD value of 0.51 for Chitwan and surrounding region based on our findings. ${ }^{15}$

\section{Statistical analysis}

The collected data were summarised, presented and analysed using the software SPSS V.20 for statistical analysis and interpreted according to frequency distribution, percentage and $\chi^{2}$ test. In statistical analysis $\mathrm{p}<0.05$ was 
considered significant. A series of figure and tables have been used to represent the data.

\section{Patient and public involvement}

Patients were not actively involved in the development of the research question but participated as interviewees. After taking informed consent, a preformed questionnaire was used to collect the demographic and healthrelated information. We presented the study results at the first International Microbiology Conference 'Man Microbes Machine, finding solution together' organised by the Nepalese Association of Clinical Microbiologists, 2019, Kathmandu, Nepal.

A study flowchart is provided as figure 1(online supplemental figure 1).

\section{RESULTS}

Prevalence of scrub typhus infection among febrile patients

Out of a total of 1797 serum samples of febrile patients, $524(29.2 \%)$ were scrub typhus positive and 1273 (70.8\%) were negative (95\% CI, 27.1-31.3).

\section{Geographical area-wise distribution of scrub typhus cases}

The majority of the scrub typhus positive cases were from Chitwan district $271(51.7 \%)$ followed by neighbouring districts like Nawalparasi, 139 (26.5\%); Gorkha, 24 $(4.6 \%)$ and Makawanpur, 16 (3.1\%).

\section{Gender-wise distribution of scrub typhus cases}

Among 1797 febrile cases, 922 were men and 875 were women. Out of 524 seropositive scrub typhus cases, 314 $(35.9 \%)$ were women and $210(22.8 \%)$ were men. The seropositivity was predominant among women compared with men with a ratio of $1.5: 1$. This study showed statistical significant association of scrub typhus cases with gender of the patients $(\mathrm{p}<0.001)$.

\section{Age-wise distribution of scrub typhus cases}

The occurrence of scrub typhus was more common among age group of 51-60 years $(37.2 \%)$ followed by $11-20$ years $(31.8 \%)$ and $31-40$ years $(30.9 \%)$. The occurrence of scrub typhus was found least, $10(13.9 \%)$ among above 70 years of age group. There was statistically significant association between age variable in the occurrence of scrub typhus $(\mathrm{p}<0.003)$ (table 1$)$.

\section{Occupation-wise distribution of scrub typhus cases}

Scrub typhus was most common among farmers, 182 $(37.8 \%)$ followed by students, $222(30.8 \%)$ and housewives, $96(28.2 \%)$. Scrub typhus was less common, 24 $(9.4 \%)$ in other occupations like self-employers, businesspeople, teachers, drivers, office workers, health workers and preschooling group. On statistical analysis, the occupational variation of scrub typhus was significantly associated $(\mathrm{p}<0.001)$ (table 2$)$.

\section{Month-wise distribution of scrub typhus cases}

The highest number of scrub typhus positive cases were found during the month of July, $60(57.7 \%)$ followed
Table 1 Age-wise distribution of scrub typhus cases

\begin{tabular}{lcrrl}
\hline $\begin{array}{l}\text { Age group } \\
\text { (years) }\end{array}$ & $\begin{array}{l}\text { Febrile } \\
\text { cases }\end{array}$ & \multicolumn{1}{c}{$\begin{array}{l}\text { Positive } \\
\text { no. (\%) }\end{array}$} & \multicolumn{1}{l}{$\begin{array}{l}\text { Negative } \\
\text { no. (\%) }\end{array}$} & P value \\
\hline $0-10$ & 299 & $82(27.4)$ & $217(72.6)$ & 0.003 \\
$11-20$ & 346 & $110(31.8)$ & $236(68.2)$ & \\
$21-30$ & 326 & $93(28.5)$ & $233(71.5)$ & \\
$31-40$ & 272 & $84(30.9)$ & $188(69.1)$ & \\
$41-50$ & 203 & $60(29.6)$ & $143(70.4)$ & \\
$51-60$ & 183 & $68(37.2)$ & $115(62.8)$ & \\
$61-70$ & 96 & $17(17.7)$ & $79(82.3)$ & \\
$\geq 71$ & 72 & $10(13.9)$ & $62(86.1)$ & \\
Total & $\mathbf{1 7 9 7}$ & $\mathbf{5 2 4}(\mathbf{2 9 . 2})$ & $\mathbf{1 2 7 3 ( 7 0 . 8 )}$ & \\
\hline & & &
\end{tabular}

by August, 105 (41.2\%); October, 152 (30.3\%) and November, 98 (30.3\%). The least number of scrub typhus positive cases were found during March, 1 (1.9\%) and April, $3(5.4 \%)$. The variation in the monthly distribution of scrub typhus was statistically significant $(\mathrm{p}<0.001)$ (table 3).

\section{Distribution of clinical manifestation in scrub typhus cases}

Fever was the most common clinical symptom (100\%) in all scrub typhus positive cases followed by anorexia (64.7\%), headache (54.6\%), abdominal pain (19.8\%), vomiting (19.3\%), lymphadenopathy $(16.6 \%)$, jaundice $(9.4 \%)$, seizures $(6.1 \%)$ and pathognomic feature like eschar $(6.5 \%)$.

\section{Laboratory findings in scrub typhus positive cases}

Haematological parameters in scrub typhus positive cases showed thrombocytopenia, $386(73.7 \%)$; lower level of haemoglobin, 246 (46.9\%); leucocytosis, 123 (23.5\%) and leucopenia, $54(10.3 \%)$. Biochemical parameters showed raised level of transaminase enzymes (alanine aminotransferase (ALT) and aspartate aminotransferase (AST)), $367(70.0 \%)$ and 399 (76.1\%), respectively; serum bilirubin, $121(23.1 \%)$ and serum creatinine, 108 $(20.6 \%)$. Majority of the patients had elevated level of AST more than the ALT (table 4).

\section{Comparison of IgM ELISA and Weil-Felix Test}

Out of a total of 1797 serum samples of febrile cases, $524(29.2 \%)$ were IgM ELISA positive and $397(22.1 \%)$ were Weil-Felix test positive. Both ELISA and Weil-Felix test positive were 224 (12.5\%). Comparatively IgM ELISA for scrub typhus is more sensitive and specific than the Weil-Felix test. In the present study, we have considered ELISA as a reference standard technique to access the sensitivity and specificity of Weil-Felix test. The sensitivity and specificity of Weil-Felix test were $42.8 \%$ and $86.4 \%$, respectively (table 5).

\section{Correlation between ELISA and Weil-Felix Test}

The correlation between IgM ELISA and Weil-Felix test showed statistically significant positive correlation $(\mathrm{r}=0.319, \mathrm{p}<0.001)$. 
Table 2 Occupation-wise distribution of scrub typhus cases

\begin{tabular}{lccc}
\hline Occupational group & Febrile cases & Positive no. (\%) & Negative no. (\%) \\
\hline Farmer & 482 & $182(37.8)$ & $300(63.1)$ \\
Student & 720 & $222(30.8)$ & $496(69.2)$ \\
Housewife & 341 & $96(28.2)$ & $245(71.8)$ \\
Others & 254 & $24(9.4)$ & $230(90.6)$ \\
Total & $\mathbf{1 7 9 7}$ & $\mathbf{5 2 4 ( 2 9 . 2 )}$ & $\mathbf{1 2 7 3 ( \mathbf { 7 0 . 8 } )}$ \\
\hline
\end{tabular}

\section{DISCUSSION}

Scrub typhus is endemic in Asia-Pacific region including tropical countries like Nepal. Post-earthquake there are increasing reports of outbreak of scrub typhus from various districts affected by earthquake. ${ }^{8}$ Scrub typhus still remains underdiagnosed due to low index of suspicion, non-specific clinical presentation, lack of diagnostic facilities and limited awareness. Therefore, scrub typhus should be considered in differential diagnosis of any febrile illness. ${ }^{16}$

The prevalence of scrub typhus varies from $0 \%-8 \%$ to $60 \%$ in different countries. ${ }^{17}$ Out of 1797 febrile cases, $524(29.2 \%)$ were found to be scrub typhus positive which was comparable with studies conducted by Narvencar $e t$ $a l^{18}(34 \%)$ and Khan et $a l^{19}(19.4 \%)$. The positivity was observed to be higher in reports from Sedhain et $a t^{20}$ $(35.9 \%)$, Munilakshmi et $a l^{21}(41.7 \%)$ and Narvencar $e t$ $a l^{18}(34 \%)$. The reason for this disparity may be attributed to non-specific presentation of the disease, sample size, geographical region and diagnostic method used.

Majority of the patients enrolled in our study were from flat lands of the country where most of them were from the rural and semi-urban areas. The majority of scrub typhus positive cases were from Chitwan district (51.7\%) followed by neighbouring districts Nawalparasi $(26.5 \%)$ and Gorkha $(4.6 \%)$. The percentage of rural participants seropositive for scrub typhus was higher than that in urban areas $(37.3 \%$ vs $22.2 \%)$. Although Chitwan was not severely affected by earthquake, the presence of scrub bushes and tropical climate must be a crucial factor for typhus prevalence.

Scrub typhus positivity was significantly higher among women, 314 (35.9\%) compared with men, 210 (22.8\%) with the ratio of 1.5:1. Likewise, Bithu et al ${ }^{22}$ Vivekanandan et $a l^{23}$ and Saleem $e t a l^{24}$ also found female preponderance in their study. In contrast, Jyothi $e t a l^{25}$ and Medhi et $a l^{26}$ found that prevalence rate for scrub typhus was higher among men. A study done by Ogawa $e t a l^{27}$ has stressed the fact that age and sex are known to influence the occurrence of scrub typhus. However, Narvencar et $a l^{18}$ did not mention any predilection for sex.

This study showed statistical significant association of scrub typhus cases with gender of the patients $(p<0.001)$. This remarkable differences observed could be associated with high exposure of women to mites while working in scrub-prone areas like agricultural fields during harvesting or cultivation of crops or jungles. On the other hand, most of the men were less exposed to mites as they were involved in indoor work as in the office, factory and company.

In this study, higher seropositivity was observed in the age group below 60 years of age, but prevalence was higher in middle-age group (51-60 years) similar to the study done in Nepal by Upadhyaya et al. ${ }^{28}$ Various authors

\begin{tabular}{|c|c|c|c|c|}
\hline Months (2016-2017) & Febrile cases & Positive no. (\%) & Negative no. (\%) & $P$ value \\
\hline July 2016 & 104 & $60(57.7)$ & 44 (42.3) & $<0.001$ \\
\hline August & 255 & $105(41.2)$ & $150(58.8)$ & \\
\hline September & 216 & $59(27.3)$ & $157(72.7)$ & \\
\hline October & 501 & $152(30.3)$ & $349(69.7)$ & \\
\hline November & 323 & 98 (30.3) & $225(69.7)$ & \\
\hline December & 88 & $19(21.6)$ & $69(78.4)$ & \\
\hline January 2017 & 34 & $9(26.5)$ & 25 (73.5) & \\
\hline February & 36 & $5(13.9)$ & $31(86.1)$ & \\
\hline March & 51 & $1(1.5)$ & $51(98.1)$ & \\
\hline April & 56 & $3(5.3)$ & $53(94.6)$ & \\
\hline May & 59 & $6(10.2)$ & 53 (89.8) & \\
\hline June & 73 & $7(9.6)$ & 66 (90.4) & \\
\hline Total & 1797 & $524(29.2)$ & $1273(70.8)$ & \\
\hline
\end{tabular}




\begin{tabular}{|c|c|}
\hline Investigations & Number (\%) \\
\hline \multicolumn{2}{|l|}{ Haematological investigation } \\
\hline Haemoglobin $(\mathrm{g} / \mathrm{L})(<110)$ & $246(46.9)$ \\
\hline TLC (per cumm) (>11000) & $123(23.5)$ \\
\hline TLC (per cumm) $(<4000)$ & $54(10.3)$ \\
\hline Platelets count (per cumm) $(<150000)$ & $386(73.7)$ \\
\hline \multicolumn{2}{|l|}{ Biochemical investigation } \\
\hline Creatinine $(\mathrm{mg} / \mathrm{dL})(>1.4)$ & $108(20.6)$ \\
\hline Bilirubin T (mg/dL) (>1.4) & $121(23.1)$ \\
\hline ALT (IU/L) (>45.0) & $367(70.0)$ \\
\hline AST (IU/L) (>40.0) & $399(76.1)$ \\
\hline
\end{tabular}

ALT, alanine aminotransferase; AST, aspartate aminotransferase; TLC, total leucocyte count.

reported scrub typhus cases in variable age groups for example, Girija et $a l^{29}$ in $13-71$ years, Singh et a $\hat{l}^{00}$ in 15-65 years. The high prevalence among middle-age group might be due to the fact that this young and active population are mostly involved in outdoor occupational activities with more exposure to scrub vegetation and bite of mites, thus the risk of the disease is higher.

In the Asia-Pacific region among rural population, scrub typhus is essentially an occupational disease. Scrub typhus was more common among farmers (37.8\%) followed by students and housewives which is almost similar to a study by Inamdar et al. ${ }^{31}$ This study shows statistically significant association between scrub typhus infection and nature of occupation $(\mathrm{p}<0.001)$. The trend is more common among people engaged in occupational or recreational behaviour. Additionally, students were from rural areas involved in both agriculture and study in schools and colleges near scrub jungles, thus, it brings them into contact with mite-prone habitats such as scrub (bush) and grass which might be the strong reason to acquire scrub typhus.

The seasonal occurrence of scrub typhus varies in different countries with climate. Although scrub typhus cases were reported throughout the year, most of them were seen from July to February of next year. Similarly various studies reported peak seasonal distribution of scrub typhus cases during monsoon and post-monsoon period that is, Kumar et al, ${ }^{32}$ July-August; Huang et al, ${ }^{33}$
June-July; Upadhyaya et al, July-November. Monsoon season is favourable for mites to lay eggs, just following post-monsoon period that coincides with growth of scrub vegetation which is a habitat for mites. Since agricultural workers are mostly involved in fields during these months, there is high risk to the bites of larval mites.

In the present study, in all cases $(100 \%)$, fever was the most common clinical symptom similar to other studies. ${ }^{34}$ It is followed by anorexia, headache, abdominal pain, vomiting, lymphadenopathy, jaundice and seizures, respectively. Though the pathognomonic of scrub typhus is eschar formation at the site of bite, it has a variable occurrence from $7 \%$ to $80 \%{ }^{35}$ However, eschar formation is rarely reported from South East Asian regions. ${ }^{23}$ In this study eschar formation was seen in only $6.5 \%$ of patients. In contrast, Ramyasree et $a l^{36}$ reported no eschar. None of these non-specific clinical presentations including eschar is diagnostic of scrub typhus. Hence, epidemiological factors pertaining to geographical area, occupation, habitat and history of travel could certainly assist in diagnosis.

During the study period, five female patients within age group 14-51 years with scrub typhus died due to multi-organ failure. Serology showed both IgM ELISA and Weil-Felix test positive. Haematological parameters revealed thrombocytopenia, lower level of haemoglobin, leukocytosis and leucopenia. Biochemical parameters revealed raised level of transaminase enzymes (ALT and AST), respectively, serum bilirubin and serum creatinine. All the patients had elevated level of AST more than the ALT. Similarly, a total of eight deaths were recorded from eastern and far western region of Nepal by Upadhyaya $e t a l$. The case fatality rate for scrub typhus has been reported up to $30.0 \%$ from different countries. ${ }^{37}$ However, the mortality rate in this study was $0.9 \%$ comparable to study by Kabi et al. ${ }^{38}$ This might be due to the growing awareness among people and timely administration of the antibiotics (doxycycline).

In this study thrombocytopenia and hepatic dysfunction were the most common complications. Most of the patients had elevated serum transaminases and serum bilirubin. Laboratory investigations revealed lower level of haemoglobin, leukocytosis and leucopenia. This finding was similar to other studies. ${ }^{39}$ The biochemical parameters showed raised level of creatinine, liver enzymes like ALT and AST. Majority of the scrub typhus positive cases

Table 5 Comparison of IgM ELISA and Weil-Felix Test

\begin{tabular}{lllrr}
\hline & & ELISA test & & \\
\cline { 3 - 5 } Description & & Positive & Negative & Total \\
\hline Weil-Felix Test & Positive & $224(12.5 \%)$ & $173(9.6 \%)$ & $\mathbf{3 9 7}(\mathbf{2 2 . 1} \%)$ \\
& Negative & $300(16.7 \%)$ & $1100(61.2 \%)$ & $\mathbf{1 4 0 0 ( 7 7 . 9 \% )}$ \\
& Total & $\mathbf{5 2 4}(\mathbf{2 9 . 2} \%)$ & $\mathbf{1 2 7 3}(\mathbf{7 0 . 8} \%)$ & $\mathbf{1 7 9 7 ( \mathbf { 1 0 0 } \% )}$ \\
\hline
\end{tabular}


showed raised level of AST more than ALT, which is in concordance with the study conducted by Jamil $e t a t^{40}$

Due to non-specific clinical presentation, common method employed for the diagnosis of scrub typhus is through serological tests. In the present study, $29.2 \%$ of serum samples of febrile cases were IgM ELISA positive and $22.1 \%$ were Weil-Felix test positive. Both ELISA and Weil-Felix test (OS-K strain) positive were $12.5 \%$. In WeilFelix test, the diagnostic titre of OS-K antigen was $\geq 160$. We proposed a cutoff-OD value of 0.51 for Chitwan and surrounding region based on our findings.

The gold standard test for scrub typhus is indirect immunofluorescence assay which is not used in this study, that would have been the best indicator to find sensitivity and specificity of other tests. However, the scrub typhus IgM ELISA is a flexible alternative to the IFA technique. It has sensitivity and specificity of more than $90.0 \%$ for detecting specific antibodies.

In the present study, we have considered ELISA as a reference standard technique to access the sensitivity and specificity of Weil-Felix test. The sensitivity and specificity of Weil-Felix test were $42.8 \%$ and $86.4 \%$, respectively. WeilFelix test findings were correlated with ELISA reports to determine its sensitivity and specificity. The correlation between IgM ELISA and Weil-Felix test showed statistically significant positive correlation $(\mathrm{r}=0.319, \mathrm{p}<0.001)$. Hence, comparatively IgM ELISA for scrub typhus is more sensitive and specific than the Weil-Felix test. Koraluru et $a l^{41}$ reported sensitivity and specificity of ELISA and WeilFelix test were $85.3 \%, 95.5 \%$ and $67.1 \%, 93.3 \%$, respectively when compared with IFA test. A study by Coleman $e t$ $a l^{42}$ reported high sensitivity and specificity of IgM ELISA. The limitation of this study is that culture technique for isolation of $O$. tsutsugamush $i$ and its molecular characterisation was not done.

The specific gold standard confirmatory tests like indirect immunoperoxidase and IFA are costly and not easily available in developing countries like Nepal. Weil-Felix test is simple and economical that can serve as a screening test in the diagnosis of scrub typhus when there is unavailability of definitive tests. ELISA is a comparatively cheaper and easier test to perform without requirement of expensive fluorescent microscopes. ELISA technique is highly sensitive and reproducible for IgM antibodies and can be used as a specific test for diagnosis of scrub typhus.

\section{Conclusion}

This study implies the emergence of scrub typhus. Although the disease is endemic in our country, it is grossly underdiagnosed owing to non-specific clinical presentations and lack of affordable and reliable diagnostic facilities. Thus, it is recommended that high index of suspicion should be maintained for cases presenting with acute febrile illness. Unfortunately, there is no effective vaccine against scrub typhus and the disease still persists. Infection with scrub typhus was found high and this calls for an urgent need to introduce vaccine against scrub typhus. Delay in diagnosis and treatment can be fatal and may lead to complications and higher mortality. Hence, early diagnosis and prompt institution of effective antibiotic therapy is mandatory. Surveillance and public health awareness about the disease transmission and preventive measures need to be initiated.

Acknowledgements The authors express their sincere gratitude to the Department of Microbiology, Chitwan Medical College Teaching Hospital (CMC-TH), Bharatpur, Nepal. We extend our sincere thanks to all faculty members and staff of CMC-TH. Our special thanks to all the patients who participated in this study and cooperated during data collection. It was a pleasure to be associated with them through this work.

Contributors ST conceived and designed the study. PH prepared the questionnaire. $\mathrm{PH}$ collected and processed the samples. JPS, PH and LBS analysed the results. ST, $\mathrm{PH}$ and NKC prepared the initial draft of the manuscript. NKC and PH searched the scientific literature. ST and LBS prepared and refined the manuscript.

Funding The authors have not declared a specific grant for this research from any funding agency in the public, commercial or not-for-profit sectors.

\section{Competing interests None declared.}

Patient and public involvement Patients and/or the public were not involved in the design, or conduct, or reporting, or dissemination plans of this research.

\section{Patient consent for publication Not required.}

Ethics approval Ethical approval was obtained from Chitwan Medical College Institutional Review Committee (CMC-IRC), Ref No: IRC 2073/74: 74 before starting the study. Informed consent was obtained from all the patients.

Provenance and peer review Not commissioned; externally peer reviewed.

Data availability statement Data are available in a public, open access repository. Data are available upon reasonable request. Extra data can be accessed via the Dryad data repository at http://datadryad.org/ with the doi: 10.5061/dryad. q2bvq83gz.

Open access This is an open access article distributed in accordance with the Creative Commons Attribution Non Commercial (CC BY-NC 4.0) license, which permits others to distribute, remix, adapt, build upon this work non-commercially, and license their derivative works on different terms, provided the original work is properly cited, appropriate credit is given, any changes made indicated, and the use is non-commercial. See: http://creativecommons.org/licenses/by-nc/4.0/.

ORCID iD

Sangita Thapa http://orcid.org/0000-0002-2026-544X

\section{REFERENCES}

1 Walker DH, Dumler JS, Marrie T. Rickettsial diseases. In: Longo DL, Fauci AS, Kasper DL, et al, eds. Harrison's principle of internal medicine. 18th edn. USA: The McGraw-Hill Companies, 2012: 1064-5.

2 Watt G. Scrub typhus. In: Warrell DA, Cox TM, Firth JD, et al, eds. Oxford textbook of medicine. 4th edn. Oxford: University Press, Oxford, 2003: 6. 629-31.

3 Poomalar GK, Rekha R. A case series of scrub typhus in obstetrics. J Clin Diagnostic Res 2014;8:OR01-3.

4 Murdoch DR, Woods CW, Zimmerman MD, et al. The etiology of febrile illness in adults presenting to Patan hospital in Kathmandu, Nepal. Am J Trop Med Hyg 2004;70:670-5.

5 McGready R, Blacksell SD, Luksameetanasan R, et al. First report of an Orientia tsutsugamushi type TA716-related scrub typhus infection in Thailand. Vector Borne Zoonotic Dis 2010;10:191-3.

6 Basnyat B, Belbase RH, Zimmerman MD, et al. Clinical features of scrub typhus. Clin Infect Dis 2006;42:1505-6.

7 Brown GW, Shirai A, Gan E, et al. Antibodies to typhus in Eastern Nepal. Trans R Soc Trop Med Hyg 1981;75:586-7.

8 The Kathmandu Post. Rats causing scrub typhus: WHO team. Available: http://kathmandupost.ekantipur.com/news/2015-10-07/ rats-causingscrub-typhus-who-team.html

9 Poudel A. Three dead and 91 infected with scrub typhus so far. My Rep, 2016. Available: www.myrepublica. com/news/3945

10 Batra HV. Spotted fevers \& typhus fever in Tamil Nadu. Indian J Med Res 2007;126:101-3. 
11 Thompson CN, Blacksell SD, Paris DH, et al. Undifferentiated febrile illness in Kathmandu, Nepal. Am J Trop Med Hyg 2015;92:875-8.

12 Nayak N. Scrub typhus in Nepal. Nepal J Epidemiol 2016;6:563-4.

13 Basnyat B. Typhoid versus typhus fever in post-earthquake Nepal. Lancet Glob Health 2016;4:e516-7.

14 PUO screen. Screening test for typhoid, brucellosis and scrub typhus fever. Tulip diagnostic $(P)$ limited

15 InBios. Scrub typhus detect IgM ELISA system [Package Insert]. Seattle, WA, USA: InBios International Inc, 2012

16 Thapa S, Sapkota LB, Hamal P. Threat of scrub typhus in postearthquake Nepal. J Chitwan Med Coll 2016;6:1-6.

17 Chanyasanha C, Kittigul L, Puenchitton S, et al. Antibodies titers to rickettsial diseases in blood donor in Bangkok by indirect immunoloperoxidase technique. Proc Natl Epidemiol Semin Bangkok 1992:167-9.

18 Narvencar KPS, Rodrigues S, Nevrekar RP, et al. Scrub typhus in patients reporting with acute febrile illness at a tertiary health care institution in Goa. Indian J Med Res 2012;136:1020-4.

19 Khan F, Mittal G, Agarwal RK, et al. Prevalence of Scrub Typhus - A Cause of concern in Uttarakhand Region, India. Int J Curr Microbiol App Sci 2015;1:101-9.

20 Sedhain A, Bhattarai GR. Clinical presentation of scrub typhus during a major outbreak in central Nepal. Asian J Med Sci 2017;8:27-31.

21 Munilakshmi P, Krishna MV, John MS, et al. FUO cases showing prevalence of scrub typhus: a comparative study by ELISA and rapid test in a tertiary care hospital in Andhra Pradesh, India. Int J Curr Microbiol App Sci 2015;4:632-40.

22 Bithu R, Kanodia V, Maheshwari RK. Possibility of scrub typhus in fever of unknown origin (FUO) cases: an experience from Rajasthan. Indian J Med Microbiol 2014;32:387-90.

23 Vivekanandan M, Mani A, Priya YS, et al. Outbreak of scrub typhus in Pondicherry. J Assoc Physicians India 2010;58:24-8.

24 Saleem M, Shivekar S, Gopal R. Clinico laboratory profiles of scrub typhus at a rural tertiary care hospital in South India. Int J Curr Res Revi 2015;7:75-80

25 Jyothi R, Sahira H, Sathyabhama MC, et al. Seroprevalence of scrub typhus among febrile patients in a tertiary care hospital in Thiruvananthapuram, Kerala. J Acad Ind Res 2015;3:542-5.

26 Medhi M, Sonowal A, Saikia L, et al. Seroprevalence of scrub typhus in a tertiary care hospital of upper Assam. Int J Med Res Prof 2016;2:24-7.

27 Ogawa M, Hagiwara T, Kishimoto T, et al. Scrub typhus in Japan: epidemiology and clinical features of cases reported in 1998. Am J Trop Med Hyg 2002;67:162-5.
28 Upadhyaya BP, Shakya G, Adhikari S, et al. Scrub typhus: an emerging neglected tropical disease in Nepal. J Nepal Health Res Counc 2016;14:122-7.

29 Girija S, Rajan A, Sathiyanarayanan J, et al. Scrub typhus- an emerging disease in South India. IJRRMS 2013;3:11-13.

30 Singh SK, Ram R, Marangmei L, et al. Case series on scrub typhus from a tertiary care hospital of North East India. IOSRJDMS 2014;13:62-4.

31 Inamdar S, Acharya R, Vijayanarayana K, et al. Study of clinical characteristics and treatment pattern of scrub typhus in tertiary care hospital. J Pharm Sci Res 2013;5:107-10.

32 Kumar R, Srinivasan P. A study of clinical and laboratory profile of scrub typhus in children in a tertiary hospital in South India. Int $J$ Contemp Pediatrics 2017;4:482.

33 Huang CT, Chi $\mathrm{H}$, Lee $\mathrm{HC}$, et al. Scrub typhus in children in a teaching hospital in eastern Taiwan, 2000-2005. South-East Asian J Trop Med Public Health 2009;40:789-94.

34 Singh S, Singh R, Ahmad N. A study of complications of scrub typhus in a tertiary health care Institute of Uttarakhand, India. Int $J$ Res Med Sci 2014;2:246-9.

35 Kim D-M, Won KJ, Park CY, et al. Distribution of eschars on the body of scrub typhus patients: a prospective study. Am J Trop Med Hyg 2007;76:806-9.

36 Ramyasree A, Kalawat U, Rani ND, et al. Seroprevalence of scrub typhus at a tertiary care hospital in Andhra Pradesh. Indian J Med Microbiol 2015;33:68-72.

37 Parola P, Raoult D. Ticks and tickborne bacterial diseases in humans: an emerging infectious threat. Clin Infect Dis 2001;32:897-928.

38 Kabi S, Das C, Rath SN, et al. Evaluation of two diagnostic methods of scrub typhus with Bayes rule. Int $J$ Adv Med 2017;4:688-92.

39 Varghese GM, Abraham OC, Mathai D, et al. Scrub typhus among hospitalised patients with febrile illness in South India: magnitude and clinical predictors. J Infect 2006;52:56-60.

40 Jamil M, Lyngrah KG, Lyngdoh M, et al. Clinical Manifestations and Complications of Scrub Typhus : A Hospital Based Study from North Eastern India. J Assoc Physicians India 2014;62:19-23.

41 Koraluru M, Bairy I, Varma M, et al. Diagnostic validation of selected serological tests for detecting scrub typhus. Microbiol Immunol 2015:59:371-4.

42 Coleman RE, Sangkasuwan V, Suwanabun N, et al. Comparative evaluation of selected diagnostic assays for the detection of IgG and IgM antibody to Orientia tsutsugamushi in Thailand. Am J Trop Med Hyg 2002;67:497-503 